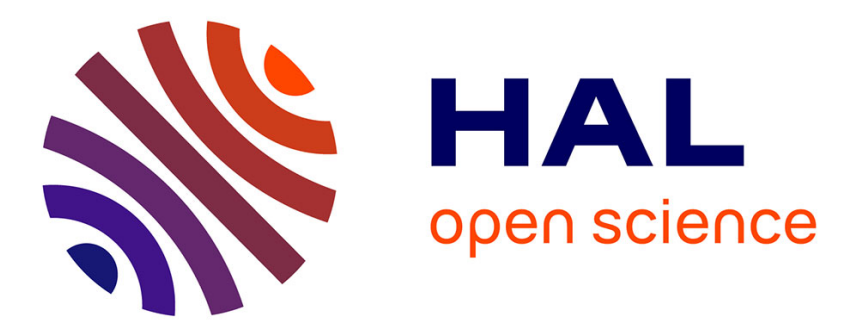

\title{
Revisiting the Nesbit and McGinnis optimization model of the golf swing hub path
}

Benjamin O’Brien, Brett Juhas, Marta Bieńkiewicz, Christophe Bourdin

\section{To cite this version:}

Benjamin O'Brien, Brett Juhas, Marta Bieńkiewicz, Christophe Bourdin. Revisiting the Nesbit and McGinnis optimization model of the golf swing hub path. Journal of Sports Medicine and Physical Fitness, 2020, 60 (8), 10.23736/S0022-4707.20.10733-3 . hal-02946879

\section{HAL Id: hal-02946879 \\ https://hal.science/hal-02946879}

Submitted on 24 Sep 2020

HAL is a multi-disciplinary open access archive for the deposit and dissemination of scientific research documents, whether they are published or not. The documents may come from teaching and research institutions in France or abroad, or from public or private research centers.
L'archive ouverte pluridisciplinaire HAL, est destinée au dépôt et à la diffusion de documents scientifiques de niveau recherche, publiés ou non, émanant des établissements d'enseignement et de recherche français ou étrangers, des laboratoires publics ou privés. 


\title{
REVISITING THE NESBIT AND MCGINNIS OPTIMISATION MODEL OF THE GOLF SWING HUB PATH
}

\author{
Benjamin O’Brien, Brett Juhas, Marta Bieńkiewicz, Christophe Bourdin \\ Aix-Marseille Univ, CNRS, ISM, Marseille, France
}

BACKGROUND: This article details the development of adopting the Nesbit and McGinnis model of the golf swing ${ }^{1}$ as a starting point for studying golf performance optimisation. The model was selected as it presents an opportunity to examine how non-naïve participants can learn and improve their swing mechanics, which could prove valuable in studying human learning in sports, rehabilitation, and re-education. METHODS: Kinematic data was acquired in laboratory and real-world environments using the motion capture systems Qualysis and CodaMotion CX-Sport, respectively. In the early stages of developing the model in MATLAB, we identified limitations in the Nesbit and McGinnis methodology, including the filtering techniques applied to swing vectors and the selection of swing variables and the solutions to their boundary conditions solutions during the downswing. By addressing these issues, our goal was to revise the model and make it more robust and capable of optimising the impact velocities from a wider variety of subjects with varying swing mechanics. RESULTS: By increasing the cutoff frequency used to filter the swing vectors and expanding the swing variable polynomial equations, we found it was possible for all participants to increase their club head velocity at impact while respecting their unique kinematic limitations. The manner of the kinematic changes and the percent of velocity improvement are participant dependent. CONCLUSIONS: Our study showed that the observed and optimised hub paths differed among participants, which suggests participants might also differ in their approaches and capacities to adopt the latter.

Keywords: golf; motion analysis; kinematics; motor control.

\section{BACKGROUND:}

A major research focus in the field of sports sciences is improving sport performance, which traditionally involves multidisciplinary knowledge, from sociology to 
psychology, biomechanics, and also neurosciences. Because of the specialty of skills required for improvement, the scientific questions are numerous and quite challenging, especially when concerning the golf swing, which is considered the most difficult motor skill in sports. ${ }^{2}$ This characterisation is due to the complex mechanics and conditions required to perform the gesture successfully and consistently. A significant amount of research has been devoted to studying and revealing the biomechanical complexities of

33 the golf swing, including studies on the role of ground-reaction forces, ${ }^{3} \mathrm{X}$-factor, ${ }^{4}$ and

34 the crunch-factor. ${ }^{6}$ A first step towards improving golf swing mechanics is to identify variables that are easily controllable by golfers and then determine the optimised trajectory of such variables.

In general, an increase in club head speed will increase the overall energy transferred to the ball at impact, which will theoretically result in a greater distance the ball travels. ${ }^{789}$ To understand and potentially optimise club head speed, there are several golf swing models, including kinematic ${ }^{10}$ and forward dynamic. ${ }^{11}$ Recent work

41 suggests that the nature of the club head path is non-circular ${ }^{12}{ }^{13}$ and derived from the path of the hands (hub path) located at the club grip.

Nesbit and McGinnis developed a forward kinematic model ${ }^{1}$ and found that,

44 while constraining the maximum force and torque outputs, participants could manipulate the hub path to increase club head velocity. Nesbit identified the hub radius,

46 hub path, and club head path ${ }^{14}$ as a collection of kinematic variables that golfers can

47 manipulate in unique ways to improve form. Figure 1 offers a two-dimensional

48 representation of these variables during the downswing as projected onto a swing plane,

49 where the hub radius can be inferred as the distance between the "Hub Rotation Center" and the "Hub Position." We selected the Nesbit and McGinnis club and optimisation

51 model as a starting point for our own development, as we believed its design, which 
accounts for three kinematic variables, might, in theory, increase the likelihood of nonnaïve participants being capable of manipulating them in ways that would optimise impact velocity and subsequently ball distance.

However, while developing the Nesbit and McGinnis club and optimisation models in MATLAB (The MathWorks, Inc.), several issues emerged that concerned the approach, design, and use of the model. First, while it was expected that the club head and hub path signals were to be filtered, given the significant speed of the downswing, it was equally necessary to have confidence in the impact velocity calculation so as to assess the degree of optimisation. Second, in order to calculate changes to the hub length and angular path, a centre point of rotation is necessary, yet its position depends on the golfer and their swing style. Finally, the kinematic response of these swing variables are modelled as time-dependent polynomial equations, however, multiple questions emerged regarding their selection and solving for their boundary conditions during the downswing.

For this study we wanted to first collect kinematic and kinetic data from a pool

67 of golfers with diverse swing styles and experiences. As discussed, there are many

68 factors that contribute to the success of a golf swing, some of which are quite

69 idiosyncratic. By developing an optimisation model that is robust enough to accommodate these features, we might be able to study how experts of a de facto complex motor task are capable of training and maintaining their swing form or possibly wish to learn and improve their mechanics. Experienced and high-level athletes often cite the relationship between their environment and their performance, so we wanted to first collect data in laboratory, which we could then use to develop our model so that it could accommodate the measurement of participants performing swings in the real world - on the driving range. By doing so, we believed we could better test the 
robustness of our club and optimisation models. After collecting our dataset, our goal was to develop an optimisation routine that uses the Nesbit and McGinnis ${ }^{1}$ model of the golf swing as a starting point. By making these changes we hypothesise that our model can enhance the findings reported in the Nesbit and McGinnis ${ }^{1}$ by optimising the impact velocity for swings produced on the driving range.

\section{METHODS:}

\section{Participants and data collection}

Four golfers (all male) were analysed for this study, and their details are given in Table 1. All participants used the same club (driver of length $=1.105 \mathrm{~m}$; mass $=0.394 \mathrm{~kg}$; club cg $\left.=0.669 ; \mathrm{I}_{\mathrm{cg}}=0.079 \mathrm{~kg} \cdot \mathrm{m}^{2}\right)$. All participants were instructed to complete a minimum of six swings that demonstrated their typical swing, which were recorded using a motion capture system and stored based personal feedback. To test the robustness of our model development, it was important that the golfers felt the measured trials represented their swing idiosyncrasies on average. Participant data was collected in two separate sessions, using different technical setups. However, like the study conducted by Nesbit and McGinnis, ${ }^{1}$ the markers used to locate club head and hub paths were similarly positioned along the long axis of the club shaft for both sessions. Our first goal was to collect golf swing kinematic data in a laboratory setting for preliminary use in developing our model. Our decision to begin data collection in the laboratory was both to follow the precedent set in Nesbit and McGinnis ${ }^{1}$ and validate any revisions to the model. Participant 1 was recorded at $300 \mathrm{~Hz}$ with an 11-camera motion tracking protocol using passive markers (Qualisys). Using the Qualisys marker placement protocol, ${ }^{15} 60$ markers (size: $19 \mathrm{~mm}$; weight: $2.5 \mathrm{~g}$ ) were placed on the body of Participant 1, and six were placed on the golf club. Participant 1 hit foam balls into a 
101 net in a competitive manner, where distance and accuracy are equally significant.

102 Because we would require a more streamlined experimental setup to measure

103 participant kinematics in real-world environments, this first step helped us identify

104 which kinematic features were important and necessary to adapt the Nesbit and

105 McGinnis swing optimisation model.

106 Following our collection and use of Participant 1 data in our initial model

107 development (see: Adaptations to Nesbit and McGinnis optimisation model),

108 Participant 2 was measured in the same laboratory setting. However, only two makers

109 were placed on his clavicle and hand, respectfully, and three markers were placed on the

110 golf club. This decision was based on our experience collecting Participant 1 swing

111 data, which made clear to us that a full body capture was not necessary, as a majority of

112 the marker measurements were either redundant or inapplicable to our model. In

113 addition, a significant amount of time was spent placing markers on Participant 1 to

114 ensure measurement accuracy, and, given our previous experiences recording the

115 movements of highly-skilled athletes, we wanted to do our best to reduce our

116 experimental setup time. Thus, for Participant 2 we were able to reduce the number of

117 markers used, which allowed us to reduce setup time and maintain confidence in our

118 measurements. Another important difference between the participants was that

119 Participant 2 hit Titleist PRO V1X balls into net in a similarly competitive manner. This

120 allowed us to study any differences dependent on ball materiality and modify our model

121 and its method for detecting ball impact.

122 A final round of data collection took place on a golf course driving range (Golf

123 Salette, Marseille, FR). By recording in a real-world environment, we wanted to ensure

124 the highest ecological validity, while preserving the highest spatio-temporal accuracy of

125 the data. In addition, by measuring and optimising highly skilled golfers performing in 
126 their natural environment, our research distinguishes itself from the study by Nesbit and

127 McGinnis, ${ }^{1}$ where kinematic data was only collected in a laboratory. An important goal

128 for our study was to take the laboratory conditions "outside," which would allow us to

129 measure participant data in a real-world environment and thus, provide a more accurate

130 measurement of participant swing idiosyncrasies. Although the use of different motion

131 capture systems is unorthodox, in order to test the robustness of our model, we opted for

132 a more flexible approach in obtaining kinematic and kinetic data. Thus, our choice of

133 motion capture system was based on environmental constraints: Laboratory Participants

134 1-2 were measured with wireless passive markers and multiple cameras (Qualysis),

135 whereas Participants 3-4, who performed on a driving range, were measured with the

136 CodaMotion CX-Sport active marker motion tracking system and scanner with outdoor

137 lighting exposure (sampling rate $200 \mathrm{~Hz}$ ). CodaMotion CX-Sport 3-D motion scanner

138 was placed $2.5 \mathrm{~m}$ from the golf ball (1.2 $\mathrm{m}$ above), and four markers were placed on the

139 golf club, which were connected and powered by a CodaMotion "drive box" (weight:

$14015 \mathrm{~g}$ ) fastened below the handgrip.

141 Participants verbally informed us which trial best represented their swing, which

142 was then used for analysis. This process of selection is consistent with previous

143 studies. ${ }^{12}{ }^{14} 16$ Like the Nesbit and McGinnis optimisation model, our analysis focuses

144 on the downswing, which is the phase of the full swing that requires the golfer to exert

145 the most energy to accelerate the club along the swing path.

\section{Adaptations to Nesbit and McGinnis optimisation model}

147 First, the model constrains the entire motion of the club onto one plane, ${ }^{1} 1017$ and, as

148 such, it is necessary to project 3D captured data onto a best-fit swing plane. However,

149 due to the complexity and speed of the golf swing motion, which can create camera and

150 scanner blind spots that hide sensor locations, some data could go unrecorded. In rare 
151 instances we used a third-order polynomial fit to estimate any missing data and create a

152 continuous signal during the downswing motion. If there were more than three

153 continuous sample points missing from the data, the swing was rejected for further

154 study. This decision was based on our efforts to boost the fidelity of the observed

155 swings, which, by doing so, would allow us to optimise swings that more closely

156 reflected the swing idiosyncrasies of each participant. This step became necessary when

157 applying the selected filtering techniques. The corrected continuous data were then

158 projected onto the swing plane. ${ }^{10}$

159 The best-fit swing planes were calculated for each participant using the club and

160 hub kinematic data. Because of the high concentration of recorded points that occur

161 early in the swing, we decided to reconstruct the club head path by using the cumulative

162 sum of the distance along the path to produce spline-fits for all three-dimensions. The

163 data points along each corrected path are equidistant in a 3-dimensional space, which

164 puts an equal weight on each point when applying a Principal Component Analysis

165 (PCA) to calculate the best-fit plane. To check the validity of our method, a Root Mean

166 Square Error (RMSE) was used to compare the observed Participant 1 club head data

167 with their reconstructed swing path, which found a $27 \%$ reduction in residual errors

168 between the observed swing path and the best-fit plane. Supplementary Figure 1

169 compares Participant 1 observed and reconstructed swing paths and Supplementary

170 Figure 2 illustrates the sizeable decrease in residual errors near impact for the

171 reconstructed swing. For a comprehensive swing analysis, we similarly reconstructed

172 the hub path motion, and, with the club head reconstruction, included it in the PCA.

173 Subsequently, an orthogonal projection of the observed data onto the swing plane is

174 applied (Figure 2). 
We required an accurate centre point for which the hub rotates to determine the hub angle and path. The radial distance from the hub to its centre of rotation is not constant, varying over time throughout the swing, and golfers with higher skill levels have demonstrated a reduction in the hub radius as the club approaches impact with the

179 ball. ${ }^{1314}$ After reviewing the data from Participants 1-2, the initial clavicle marker 180 position served as a feasible centre point of rotation and a good representation of the hub motion centre point. However, we did not measure the clavicle position for Participants 3-4, as the inclusion of an additional marker would have greatly reduced the sampling rate of our motion capture, which, in turn, would have affected the

184 accuracy of club head speed measurements. To address this absence, a regression model

185 was developed using the kinematic data of Participant 2, who had a height similar to

186 Participants 3-4, which was then used to estimate the clavicle initial position for the 187 remaining participants. To confirm the validity of this approach, multiple simulations were performed on Participants 1-2 data, where their clavicle markers were moved around $3 \mathrm{~cm}$ from its origin. RMSE calculations were then conducted which found there

190 was little to no effect on the calculation of impact velocity.

191 Confidence in the observed club head velocity was required if our goal was to maximise it. We identified the sampling rate and the type of ball as significant factors that contribute to adequately measure the impact velocity. Following an empirical

194 process of experimentation, we found that the data acquisition sampling rate cannot be

195 lower than $200 \mathrm{~Hz}$ given the speed and limited duration of the downswing

196 (approximately 250 milliseconds). Second, comparing the data of Participant 1 (foam 197 ball) with Participants 2-4 (standard golf ball), we observed that the former did not have 198 a large spike in the data at impact, which was expected due to club head-ball ball impact 199 and momentum transfer (Figure 3). The presence of such a dramatic change in velocity 
at impact necessitated a modified filtering technique to maintain this large velocity delta and retain the peak impact velocity. Thus, we decided to apply filtering to swing vectors up to the impact point.

Although club head and hub position data recorded from both acquisition systems appeared smooth and continuous, minor perturbations were amplified after calculating the first and second derivatives in order to get velocity and acceleration signals. Nesbit and McGinnis ${ }^{1}$ smoothed three-dimensional marker triad paths with a Butterworth low-pass filter at $6 \mathrm{~Hz}$. Coleman and Rankin used a fourth-order Butterworth reverse filter with a cutoff frequency of $10 \mathrm{~Hz}$ for the body landmarks and $20 \mathrm{~Hz}$ for the club grip, bottom of the club shaft, club head centre and ball. Table 2

210 illustrates the significant effects of changing the filter cutoff frequency on impact

211 velocity by comparing participant observed impact velocities with a fourth-order

212 Butterworth filter with cutoff frequencies at 6, 10, 15, and $20 \mathrm{~Hz}$. For our model, we

213 decided on a fourth-order Butterworth with a cutoff frequency $20 \mathrm{~Hz}$, as it did not

214 substantially dilute the true impact velocity.

215 Nesbit and McGinnis ${ }^{1}$ modelled the downswing time-histories of the three

216 kinematic variables as polynomial equations. During swing optimisation the torque,

217 force, power, and work are simulated by using these polynomial models, such that their

218 coefficients can be modified in ways that satisfy the conditions for peak force, torque,

219 and power outputs. They selected fourth-order polynomials to model the angular

220 positions of the hub (1) and club head (2) and a sixth-order polynomial for the hub

221 radius (3). However, when participant data were applied to this modelling, several issues became apparent, which required model alterations to ensure the robustness needed to

224 provide adequate results for our entire pool of participants analysed. 
First, ahead of optimisation, it was of interest to attempt to perfectly match the observed

hub and club path with a modelled polynomial fit. While the polynomial orders appear

to be selected because of how well they fit the time histories of the kinematic variables,

no reason is given. After observing the poor fit of using a fourth-order polynomial

Deviation (NRMSD) analysis was applied to calculate the error between each variable and its polynomial approximation. Table 3 shows NRMSD for club head and hub paths fitted to fourth- and fifth-order polynomial equations for Participants 1-4. These observations led us to expand the order of all polynomials by one, as defined in Equations (4)-(6).

Second, Nesbit and McGinnis assume that at the start of the downswing (time $t=0$ seconds), the hub and club head velocities are 0 , where $\dot{\theta}(0)=\dot{\theta}_{0}$ and $\dot{\gamma}(0)=\dot{\gamma}_{0}$. However, we observed that this was not the case for some participants, who appeared to move their hands and wrists as they transition from their backswing to their downswing. Instead of making this assumption, we actually assign these variables to the observed values, which, by doing so, reduce the overall disruption to the participants primary swing characteristics. Thus, when we solve the boundary conditions for our set of expanded polynomials, instead of simply setting $\dot{\theta}_{0}$ and $\dot{\gamma}_{0}$ equal zero, the initial velocity for each participant is set as the downswing begins. Although this added a level of complexity when solving for the boundary conditions for the polynomial equations, it yielded a more valid process. By expanding to the fifth-order polynomial equation and 
applying the observed initial club and hub velocities, the final club head impact velocity of the modelled swing improved dramatically. When seeding the polynomial expressions using the observed swing data, the final impact velocity error (modelled vs. observed) improved from $6.96 \%$ to $0.18 \%$.

To solve, we required the first and second derivatives of the angular position of the hub $\theta(4)$, which yielded the hub angular velocity $\dot{\theta}(7)$ and acceleration $\ddot{\theta}(8)$, respectfully.

Substituting our boundary conditions into (4), (7)-(8) yielded the expressions for constants $C_{0}$ though $C_{5}$ per approach created problems with our analysis of participant data. In particular, given the angle polynomial was amplified when transformed into the time domain. Prior to our decision to expand the order of the polynomials, we wanted to assess the accuracy of 
our poly-fit by conducting RMSE calculations, where the hub radius was expressed as both a function of the hub path and time. Using Participant 1 data, we found that by expressing the hub radius as function of time, we improved the poly-fit error from $5.55 \%$ to $0.59 \%$. Supplementary Figure 3 illustrates our process of experimenting with different polynomial orders and their effect on fit. Following this we chose to express our hub radius as a seventh-order polynomial function of time (15).

As a last step we wanted to compare our optimisation results with the Nesbit and McGinnis optimisation model, and used Participant 1 data for our comparison. As previously discussed, Nesbit and McGinnis used a Butterworth low-pass filter with a 6 Hz cutoff frequency, however, Supplementary Figure 4 illustrates how such a low frequency creates artefacts in the data, which can lead to incongruences between peak and impact velocities. Like our model, we decided to similarly filter with a $20 \mathrm{~Hz}$ cutoff frequency to have an accurate comparison between model optimisations. However, we did not employ our method of filtering up to the impact point, which, in turn, yielded different kinematic and kinetic values (see Table 4).

\section{Results}

303 The kinematic and kinetic quantities for each participant during their observed

304 downswings are provided in Table 4. The results of maximising impact velocity for all participants are given in Table 5. For Tables 4-5, Participant \#1a refers to the Nesbit and McGinnis optimisation method, whereas Participant \#1b shows the results of our optimisation model. The results for all kinetic quantities are the maximum values occurring during the downswing and are given as a percentage of the observed swing 
maximum value. Using our optimisation model: Figure 5 compares Participant 1

310 observed and optimised hub and club path trajectories; Figure 6 compares Participant 1

311 observed and optimised force, torque, power, and work responses; and Figure 7

312 compares the observed and optimised hub path for Participants 1-4.

314 Discussion and Implications

$315 \quad$ Swing and optimisation observations

316 The four participants presented diverse swing kinematic and kinetic characteristics (see

317 Table 4). The range of the kinematic and kinetic quantities among the participants was

318 considerable and quantitatively emphasised the difference in their swing mechanics and

319 club trajectories. Thus, like the study by Nesbit and McGinnis, ${ }^{1}$ we similarly captured a

320 diverse set of swing styles from a mechanics point of view.

321 While we did not have the opportunity to have the same participants test in both

322 experimental settings, we believe inter-participant variability associated with the

323 performance of such a familiar, yet difficult, task might have yielded vastly different

324 observed and optimised swings. In turn, this might give the impression that swings

325 performed in a particular experimental setting might lend themselves to greater

326 possibilities of improvement. This of course was not our goal, and one way we see our

327 study as distinguishing itself from Nesbit and McGinnis, ${ }^{1}$ was our goal to measure and

328 analyse swings performed in the real world. This was done by not only collecting

329 kinematic data associated with swings taken on an actual driving range with real balls,

330 but also developing a method for filtering the data in a way that preserved swing

331 idiosyncrasies. As we noted, we observed a significant difference concerning the use of

332 foam balls (Participant 1) versus actual golf balls (Participants 2-4). Thus the data

333 collected from Participants 1-2 served our development of the model rather than a mode

334 for which to compare against Participants 3-4. 

similar force, power, and work profiles. However we saw large differences in the torque response, which was observed during most of optimisations. Additional optimisation analysis could be conducted that further constrains each profile to better match the swing observations. Nesbit and McGinnis only constrained the torque based on the peak value, and our model similarly satisfied this constraint.

More generally, we adhered to Nesbit's constraints on applied forces, torque, and power, but slightly deviated from their approach when optimising the hub path. To reduce disruptions to the participant natural swing, we constrained the starting and ending point of the hub path, while Nesbit and McGinnis only constrain the end point to maintain proper impact with the ball. This starting point boundary condition creates

346 slightly obscure initial hub motion during the downswing. Although it presented minor

347 issues, to our mind, allowing for an unconstrained start point remedies slight disruptions 348 in the hub motion. modelled swing to try to best replicate the observed kinematic data. This would be used as a check to test the model's validity during formulation. The data-matching capability in the model was highly desired and used as a target for all incremental changes made to the original Nesbit model. ${ }^{14}$ Adhering to this goal required a certain level of controllability in model's polynomial equations, which were a bit lacking originally.

355 Changing the order of the equations, accounting for non-zero initial conditions, and 356 applying the observed initial and final hub and club angles led to similar replications of 357 the original data and large improvements in the peak impact velocity during optimisation. These changes more than likely lead to our model showing a greater club head velocity optimisation (109.37\%) when compared to our implementation of the 
360 Nesbit and McGinnis model (105.76\%). When compared side-by-side, the remaining

361 results are similar, which suggests Participant 1 has the capacity to optimise club head

362 velocity at impact, and the potential for optimisation is greater when adjustments

363 recommended by our model are made.

364 When implementing such an optimised swing in practice as a teaching

365 technique, it was believed to be advantageous to start with the natural swing kinematics

366 of the participant, and gradually make incremental changes towards the optimisation

367 target swing. This would potentially reduce any adverse effects of abrupt disruption in

368 the participant's natural swing mechanics during training.

\section{Practice implications}

371 In general, to improve and optimise golf swing mechanics, golf instructors and trainers

372 teach to the physical and psychological constraints of their pupils. However, the use of

373 motion capture systems that can visualise and make more palpable complex kinematic

374 variables only serves to inform golfers of a knowledge of results. This approach appears

375 insufficient given the complexity of activities involved in the swing.

376 Unfortunately we were unable to complete a follow-up test to examine whether

377 participants were able to use information based on hub radius, hub path, and club head

378 path optimisations to manipulate their movements. But given the complexity and

379 oftentimes subtlety of movement optimisations, which together span a short duration,

380 we imagine it might be quite difficult for them. In this case, participants would be asked

381 to not only complete a task in a way that was unnatural or counter-intuitive to their

382 previous trainings, but also to use a vocabulary of swing parameters that was most

383 likely un-familiar. One possible solution to convey this abstract information might be to

384 develop a method of augmented reality that uses artificial feedback - visual, auditory,

385 haptic, or multimodal. 
As a primary focus for improvement in golf is kinematics, Keogh and Hume ${ }^{18}$

387 theorised that different visual feedback strategies based on errors of performance might

388 help movement development. A similar approach might prove to be particularly useful

389 when comparing observed swings to those developed by an optimisation model. The

390 assumed difference between them might then be delivered to users via sensory cues.

391 Sigrist et al. ${ }^{19}$ reviewed numerous studies that examined the effects of visual, auditory,

392 haptic, or multimodal information on motor learning, some of which found significant

393 results. As there continues to be scientific evidence that the use of augmented realties

394 can influence human movement, their use for performance optimisation presents a

395 potentially exciting area for development.

396 However, the development and use of a performance optimisation tool should

397 consider the inter-individual differences in speed in which humans master a new motor

398 skill. A study by Wu et al. ${ }^{20}$ demonstrated a link between movement-to-movement

399 variability and motor learning in novice participants. The experiential exploration of

400 different movement parameters allows humans to fine-tune newly acquired actions and

401 account for motor predispositions.

402 This of course differs among individuals, given the intrinsic properties of their

403 motor system, which raises two, among many, questions. The first concerns whether the

404 repetition of an optimised movement path is the best approach for improvement, as it

405 does not provide a natural avenue for movement variability exploration, which is more

406 inline with environmental demands and the empirical constraints of the individual.

407 Several studies have shown strength and conditioning exercises have a positive effect

408 on the swing mechanics, ${ }^{21} 22$ which, by broadening the types of movement, reduces the

409 possibilities of overuse and strain. 
411 variability in terms of deviation from the optimal path cannot be standardised due to the

412 unique demands of each golfer. Not only does the amount of time to learn a new motor

413 skill differ among people, but also the threshold in which movements are perceived as

414 different. Coupling this point with the previous, it becomes clear that the use of such an

415 optimisation tool requires the attention of a golf trainer or instructor to advise and assess

416 golfer progress.

\section{Conclusion}

419 The objective of this study was to adopt the Nesbit and McGinnis model of the golf

420 swing as a point of departure for which to study golf performance optimisation. Our

421 primary goal of development was to maximise club head velocity at impact. It was also

422 of interest to observe how factors such as material and environment might affect the

423 data collected for our study. By analysing these factors, we might develop the

424 robustness of our model, which could improve future work examining how humans can

425 learn and improve complex motor tasks like the golf swing.

426 Similarly to the results reported in Nesbit and McGinnis, ${ }^{1}$ we found there was

427 potential for participant to increase their impact velocity while respecting their

428 kinematic limits. Moreover, as we hypothesised, our modifications to the model made it

429 possible to collect and use swing data from a group of participants with diverse swing

430 styles in a variety of measurement and performance environments. In review, these

431 modifications included improved filtering of swing data around impact point, increasing

432 the order of the polynomial equations used to model the three swing parameters, and

433 setting and solving the boundary coefficients to observed swings. 
behaviours of each golfer and how they affect club head velocity at impact. We showed

436

437

that the observed and optimised hub paths differed among participants, and we might assume participants might similarly differ in their capacities of adopting the latter. For this reason the development of a sensory guidance tool might aid their learning process.

Acknowledgements: This work was funded by the French National Research Agency (ANR) under the SoniMove: Inform, Guide and Learn Actions by Sounds project (ANR-14-CE24-0018-01).

\section{References}

${ }^{1}$ Nesbit S, McGinnis, R. Kinetic Constrained Optimisation of the Golf Swing Hub Path. J Sports Sci Med:2014;13:859-873.

${ }^{2}$ Dillman C, Lange G. How has biomechanics contributed to the understanding of the golf swing? Proceedings of the 1994 World Scientific Congress of Golf:1994:3-13.

${ }^{3}$ Hume P, Keogh J, Reid, D. The role of biomechanics in maximizing distance and accuracy of golf shots. J Sports Med:2005;35:429-449.

${ }^{4}$ Lamb P, Pataky T. The role of pelvis-thorax coupling in controlling within-golf club swing speed. J Sports Sci:2005;36(19):1-8.

${ }^{5}$ Myers J, Lephart S, Tsai Y, Sell T, Smoliga J, Jolly, J. The role of upper torso and pelvis rotation in driving performance during the golf swing. J Sports

Sci:2008;26(2):181-188.

${ }^{6}$ Joyce C. The most important "factor" in producing club head speed in golf. Hum Mov Sci:2017;55:138-144.

${ }^{7}$ Gordon B, Moir G, Davis S, Witmer C, Cummings,D. An investigation into the relationship of flexibility, power, and strength to club head speed in male golfers. $\mathrm{J}$ Strength Cond Res:2009;23:606-1610.

\section{${ }^{8}$ Keogh J, Marnewick M, Maulder P, Nortje J, Hume P, Bradshaw E. Are} anthropometric, flexibility, muscular strength, and endurance variables related to club head velocity in low- and high-handicap golfers? J Strength Cond Res:2009;23:18411850 .

${ }^{9}$ Sprigings E, Neal R. An insight into the importance of wrist torque in driving the golf ball: A simulation study. J Appl Biomech:2000;16:356-366. 
${ }^{10}$ Coleman S, Rankin A. A three-dimensional examination of the planar nature of the golf swing. J Sports Sci:2005;23:227-234.

${ }^{11}$ Mackenzie S, Sprigings E. A three-dimensional forward dynamics model of the golf swing. Sports Eng:2009;11(14):165-175.

${ }^{12}$ Nesbit S, McGinnis R. Kinematic Analyses of the Golf Swing Hub Path and its Role in Golfer/Club Kinetic Transfers. J Sports Sci Med:2009;8(2):235-246.

${ }^{13}$ Miura K. Parametric acceleration - The effect of inward pull of the golf club at impact stage. Sports Eng:2001;4(2):74-86.

${ }^{14}$ Nesbit S. A Three Dimensional Kinematic and Kinetic Study of the Golf Swing. J Sports Sci Med:2005;4:499-519.

${ }^{15}$ Sint Jan S. Color Atlas of Skeletal Landmark Definitions. Guidelines for Reproducible Manual and Virtual Palpations. Edinburgh: Churchill Livingstone; 2007.

${ }^{16}$ Nesbit S, Serrano M. Work and power analysis of the golf swing. J Sports Sci Med:2009;4(4):520-533.

${ }^{17}$ Kane T, Likin P, Levinson D. Spacecraft dynamics. New York: McGraw-Hill Co; 1983.

${ }^{18}$ Keogh J, Hume P. Practice conditions: How do they influence motor learning in golf? Proceedings of the $30^{\text {th }}$ Annual Conference of Biomechanics in Sports, 2012:367-370.

${ }^{19}$ Sigrist R, Rauter G, Riener R, Wolf P. Augmented visual, auditory, haptic, and multimodal feedback in motor learning: a review. Psychon Bull Rev:2013;20(1):21-53.

${ }^{20}$ Wu H, Miyamoto Y, Castro L, Olveczky B, Smith M. (2014) Temporal structure of motor variability is dynamically regulated and predicts motor learning ability. Nat Neurosci:2014;17(2):185-211.

${ }^{21}$ Smith C, Callister R, Lubans D. A systematic review of strength and conditioning programmes designed to improve fitness characteristics in golfers. J Sports Sci:2011;29(9):933-43.

${ }^{22}$ Thompson C, Cobb K, Blackwell J. Functional training improves club head speed and functional fitness in older golfers. J Strength Cond Res:2007;21(1):131-7. 
522 Table 1. Participant data.

523

\begin{tabular}{|c|c|c|c|c|c|c|}
\hline Participant & Age & Height & Weight & Handicap & Experience (yrs) & Round per Year \\
\hline 1 & 45 & 175 & 73 & 10 & 20 & 25 \\
\hline 2 & 33 & 185 & 79 & 18 & 19 & 8 \\
\hline 3 & 49 & 182 & 72 & 15 & 35 & 22 \\
\hline 4 & 23 & 186 & 72 & 2 & 11 & 42 \\
\hline
\end{tabular}

524 525 526

Table 2. Participant impact velocities $(\mathrm{m} / \mathrm{s})$ and percentage of error $(\%)$ from the observed impact velocity: observed, fourth-order Butterworth filter with cutoff frequencies at $6,10,15,20 \mathrm{~Hz}$.

\begin{tabular}{|c|c|c|c|c|c|c|}
\hline Participant & Units & $\begin{array}{c}\text { Unfiltered } \\
(\mathbf{m} / \mathbf{s})\end{array}$ & $\begin{array}{c}\mathbf{6 ~ H z} \\
(\mathbf{m} / \mathbf{s})\end{array}$ & $\begin{array}{c}\mathbf{1 0 ~ H z} \\
(\mathbf{m} / \mathbf{s})\end{array}$ & $\begin{array}{c}\mathbf{1 5} \mathbf{~ H z} \\
(\mathbf{m} / \mathbf{s})\end{array}$ & $\begin{array}{c}\mathbf{2 0 ~ H z} \\
(\mathbf{m} / \mathbf{s})\end{array}$ \\
\hline \multirow{2}{*}{ Participant \#1 } & Impact velocity & 44.63 & 35.67 & 42.77 & 44.32 & 44.57 \\
\cline { 2 - 7 } & Error \% & $0.00 \%$ & $-20.08 \%$ & $-4.17 \%$ & $-0.69 \%$ & $-0.13 \%$ \\
\hline \multirow{2}{*}{ Participant \#2 } & Impact velocity & 36.68 & 32.93 & 35.94 & 36.57 & 36.68 \\
\cline { 2 - 7 } & Error \% & $0.00 \%$ & $-10.22 \%$ & $-2.02 \%$ & $-0.30 \%$ & $0.00 \%$ \\
\hline \multirow{2}{*}{ Participant \#3 } & Impact velocity & 46.29 & 38.39 & 44.01 & 45.96 & 46.28 \\
\cline { 2 - 7 } & Error \% & $0.00 \%$ & $-17.07 \%$ & $-4.93 \%$ & $-0.71 \%$ & $-0.02 \%$ \\
\hline \multirow{2}{*}{ Participant \#4 } & Impact velocity & 54.53 & 42.11 & 50.76 & 53.93 & 54.43 \\
\cline { 2 - 7 } & Error \% & $0.00 \%$ & $-22.78 \%$ & $-6.91 \%$ & $-1.10 \%$ & $-0.18 \%$ \\
\hline
\end{tabular}

527

Table 3. NRMSD for club head and hub paths fitted to fourth- and fifth-order

polynomial equations for Participants 1-4.

\begin{tabular}{|c|c|c|c|c|}
\hline Participant & $\begin{array}{c}\text { Swing } \\
\text { variable }\end{array}$ & Unit & $\begin{array}{c}\text { Fourth-order } \\
\text { polynomial }\end{array}$ & $\begin{array}{c}\text { Fifth-order } \\
\text { polynomial }\end{array}$ \\
\hline \multirow{3}{*}{ Participant \#1 } & \multirow{3}{*}{ Club head path } & Position & $5.52 \%$ & $0.46 \%$ \\
\cline { 3 - 5 } & \multirow{3}{*}{ Hub path } & Velocity & $10.31 \%$ & $1.18 \%$ \\
\cline { 3 - 5 } & & Acceleration & $25.87 \%$ & $6.34 \%$ \\
\cline { 3 - 5 } & & Position & $5.82 \%$ & $5.07 \%$ \\
\cline { 3 - 5 } & \multirow{3}{*}{ Participant \#2 } & Velocity & $17.96 \%$ & $12.82 \%$ \\
\cline { 3 - 5 } & \multirow{3}{*}{ Club head path } & Acceleration & $24.59 \%$ & $19.04 \%$ \\
\cline { 3 - 5 } & & Position & $12.02 \%$ & $4.77 \%$ \\
\cline { 3 - 5 } & & Velocity & $27.11 \%$ & $9.80 \%$ \\
\cline { 3 - 5 } & \multirow{3}{*}{ Hub path } & Acceleration & $30.12 \%$ & $14.79 \%$ \\
\cline { 3 - 5 } & & Position & $3.73 \%$ & $0.80 \%$ \\
\cline { 3 - 5 } & & Velocity & $9.62 \%$ & $4.16 \%$ \\
\cline { 3 - 5 } & \multirow{3}{*}{ Club head path } & Acceleration & $14.27 \%$ & $11.45 \%$ \\
\cline { 3 - 5 } & Position & $5.06 \%$ & $1.26 \%$ \\
\hline & & Velocity & $10.90 \%$ & $4.70 \%$ \\
\hline
\end{tabular}




\begin{tabular}{|l|c|c|c|c|}
\hline Participant \#3 & & Acceleration & $21.70 \%$ & $16.93 \%$ \\
\cline { 3 - 5 } & \multirow{3}{*}{ Hub path } & Position & $4.04 \%$ & $5.77 \%$ \\
\cline { 3 - 5 } & & Velocity & $14.68 \%$ & $14.56 \%$ \\
\cline { 3 - 5 } & & Acceleration & $27.88 \%$ & $25.62 \%$ \\
\hline \multirow{3}{*}{ Participant \#4 } & Position & $2.94 \%$ & $0.61 \%$ \\
\cline { 3 - 5 } & \multirow{3}{*}{ Club head path } & $5.93 \%$ & $2.98 \%$ \\
\cline { 3 - 5 } & & Velocity & $17.30 \%$ & $16.16 \%$ \\
\cline { 3 - 5 } & & Acceleration & $7.28 \%$ & $3.01 \%$ \\
\cline { 3 - 5 } & \multirow{3}{*}{ Hub path } & Position & $21.34 \%$ & $9.20 \%$ \\
\cline { 3 - 5 } & & Velocity & $31.80 \%$ & $17.81 \%$ \\
\cline { 3 - 5 } & Acceleration & & \\
\hline
\end{tabular}

530

531 Table 4: Participant data observed during downswing - all values are time of maximum.

\begin{tabular}{|c|c|c|c|c|c|c|}
\hline Data Type & Units & $\begin{array}{c}\text { Participant } \\
\mathbf{\# 1 a}\end{array}$ & $\begin{array}{c}\text { Participant } \\
\mathbf{\# 1 b}\end{array}$ & $\begin{array}{c}\text { Participant } \\
\mathbf{\# 2}\end{array}$ & $\begin{array}{c}\text { Participant } \\
\mathbf{\# 3}\end{array}$ & $\begin{array}{c}\text { Participant } \\
\mathbf{4 4}\end{array}$ \\
\hline Club Head Vel & $\mathrm{m} / \mathrm{s}$ & 44.00 & 44.53 & 36.79 & 45.28 & 54.67 \\
\hline Swing Torque & $\mathrm{N} . \mathrm{m}$ & 44.66 & 47.5 & 20.35 & 51.61 & 68.03 \\
\hline Mag of Force & $\mathrm{N}$ & 446.56 & 467.85 & 313.59 & 413.67 & 585.62 \\
\hline Total Work & N.m & 392.42 & 395.16 & 243.65 & 372.93 & 589.78 \\
\hline Linear Work & $\mathrm{N} . \mathrm{m}$ & 235.21 & 234.5 & 170.9 & 241.27 & 376.56 \\
\hline Angular Work & N.m & 157.21 & 160.66 & 72.75 & 131.66 & 213.22 \\
\hline Total Power & N.m/s & 4174.72 & 4211.09 & 2167.68 & 4987.92 & 7676.41 \\
\hline Linear Power & N.m/s & 2503.78 & 2474.8 & 1614.16 & 3063.52 & 4964.34 \\
\hline Angular Power & N.m/s & 1746.75 & 1879.62 & 640.58 & 1929.95 & 3075.74 \\
\hline
\end{tabular}
532

533 Table 5: Optimisation data for all participants.

\begin{tabular}{|c|c|c|c|c|c|}
\hline Data Type & $\begin{array}{c}\text { Participant } \\
\mathbf{\# 1 a} \\
\text { Percent (\%) }\end{array}$ & $\begin{array}{c}\text { Participant } \\
\mathbf{\# 1 b} \\
\text { Percent (\%) }\end{array}$ & $\begin{array}{c}\text { Participant } \\
\mathbf{\# 2} \\
\text { Percent (\%) }\end{array}$ & $\begin{array}{c}\text { Participant } \\
\mathbf{\# 3} \\
\text { Percent (\%) }\end{array}$ & $\begin{array}{c}\text { Participant } \\
\mathbf{\# 4} \\
\text { Percent (\%) }\end{array}$ \\
\hline Club Head Vel & 105.76 & 109.37 & 105.63 & 108.30 & 107.70 \\
\hline Swing Torque & 99.84 & 93.18 & 99.77 & 96.51 & 99.98 \\
\hline Mag of Force & 95.52 & 92.53 & 92.96 & 93.14 & 97.00 \\
\hline Total Work & 123.72 & 105.36 & 106.84 & 120.55 & 123.77 \\
\hline Linear Work & 125.79 & 128.14 & 119.71 & 126.75 & 134.07 \\
\hline Angular Work & 120.62 & 72.10 & 76.60 & 109.20 & 105.59 \\
\hline Total Power & 83.86 & 81.46 & 83.68 & 86.26 & 89.63 \\
\hline Linear Power & 99.99 & 99.88 & 99.84 & 99.80 & 99.47 \\
\hline Angular Power & 94.26 & 98.12 & 56.97 & 93.47 & 91.06 \\
\hline
\end{tabular}




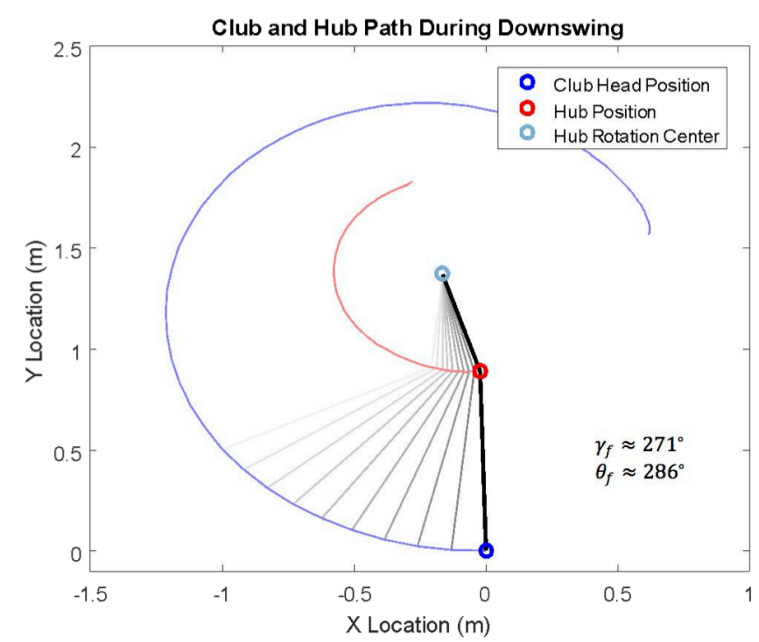

539 Figure 1. Illustration of the hub radius, hub path, and club head path during the 540 downswing as projected onto a 2-dimensional swing plane.

541

542

546

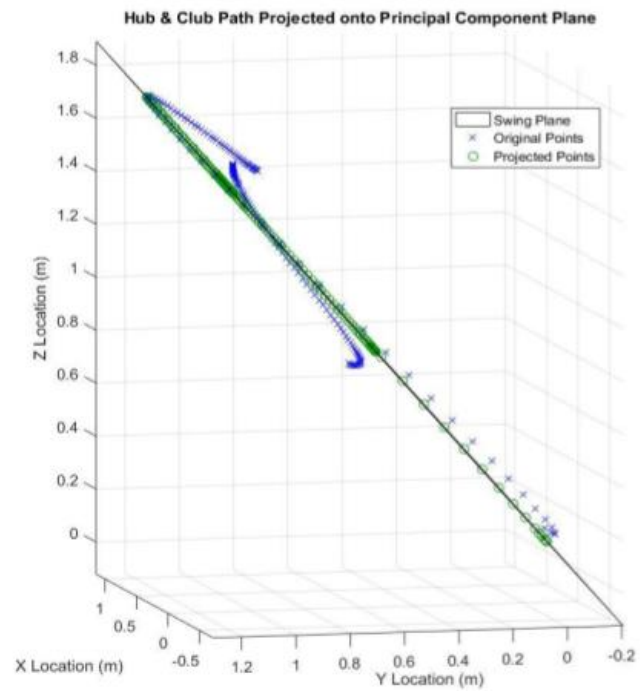

Figure 2. Participant 1 original (blue) and projected (green) club head and hub paths projected onto swing plan. The two plots show different $3 \mathrm{D}$ orientation views of the same swing data. 

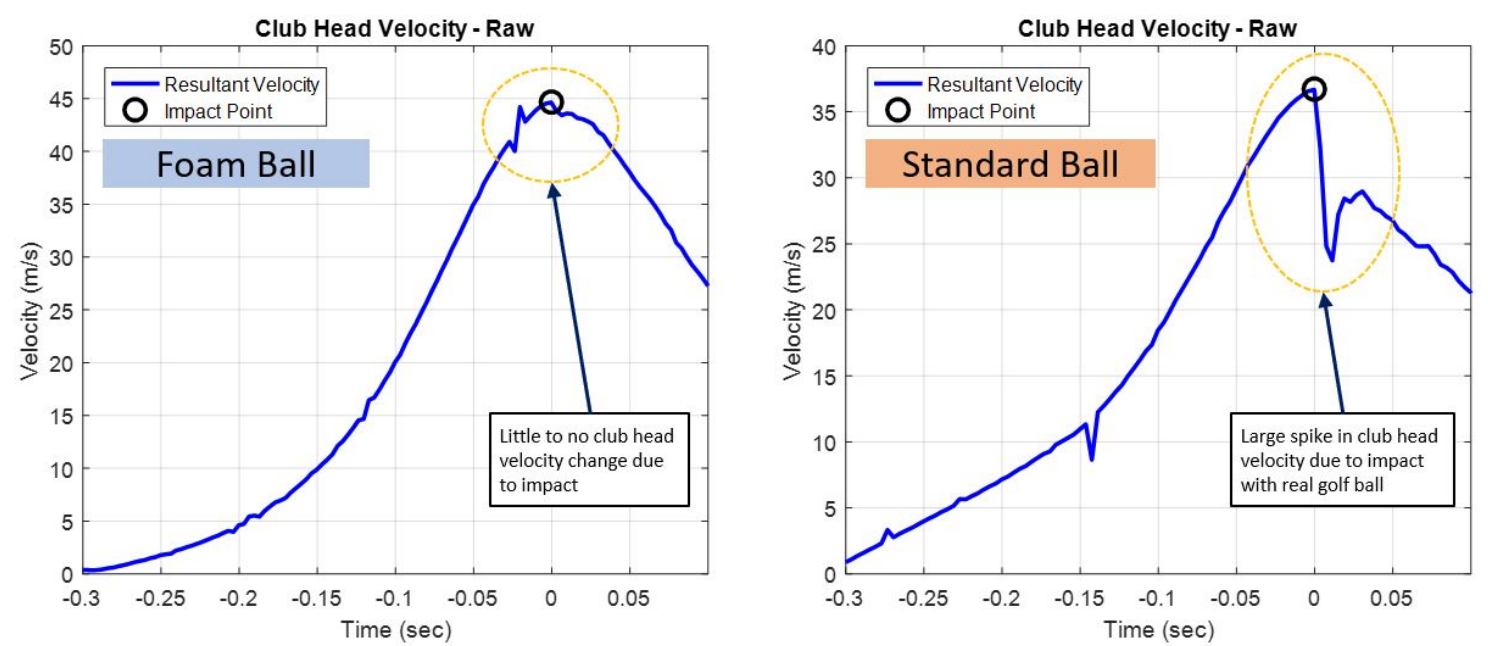

Figure 3. Club head raw resultant velocity comparison, during impact, for Participant 1 hitting a foam ball (left) and Participant 2 (right) hitting a real standard golf ball.
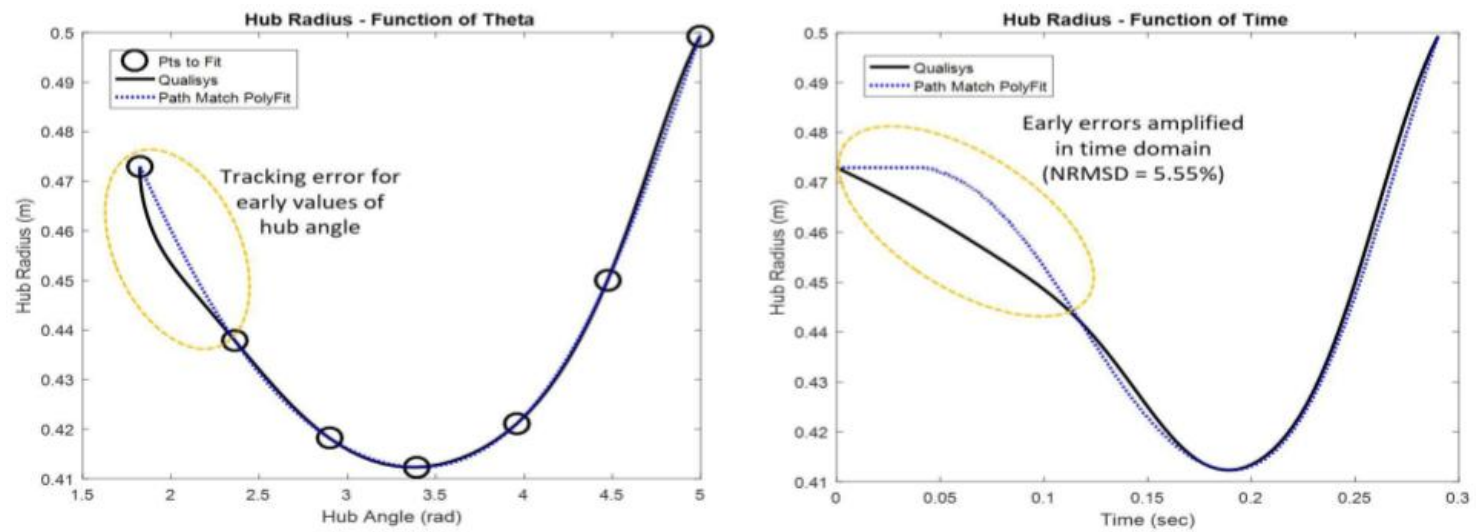

553 Figure 4: Participant 1 hub radius as a function of hub angular position (Left) and the corresponding hub radius as a function of time (Right) showing amplified errors transformed into the time domain. 

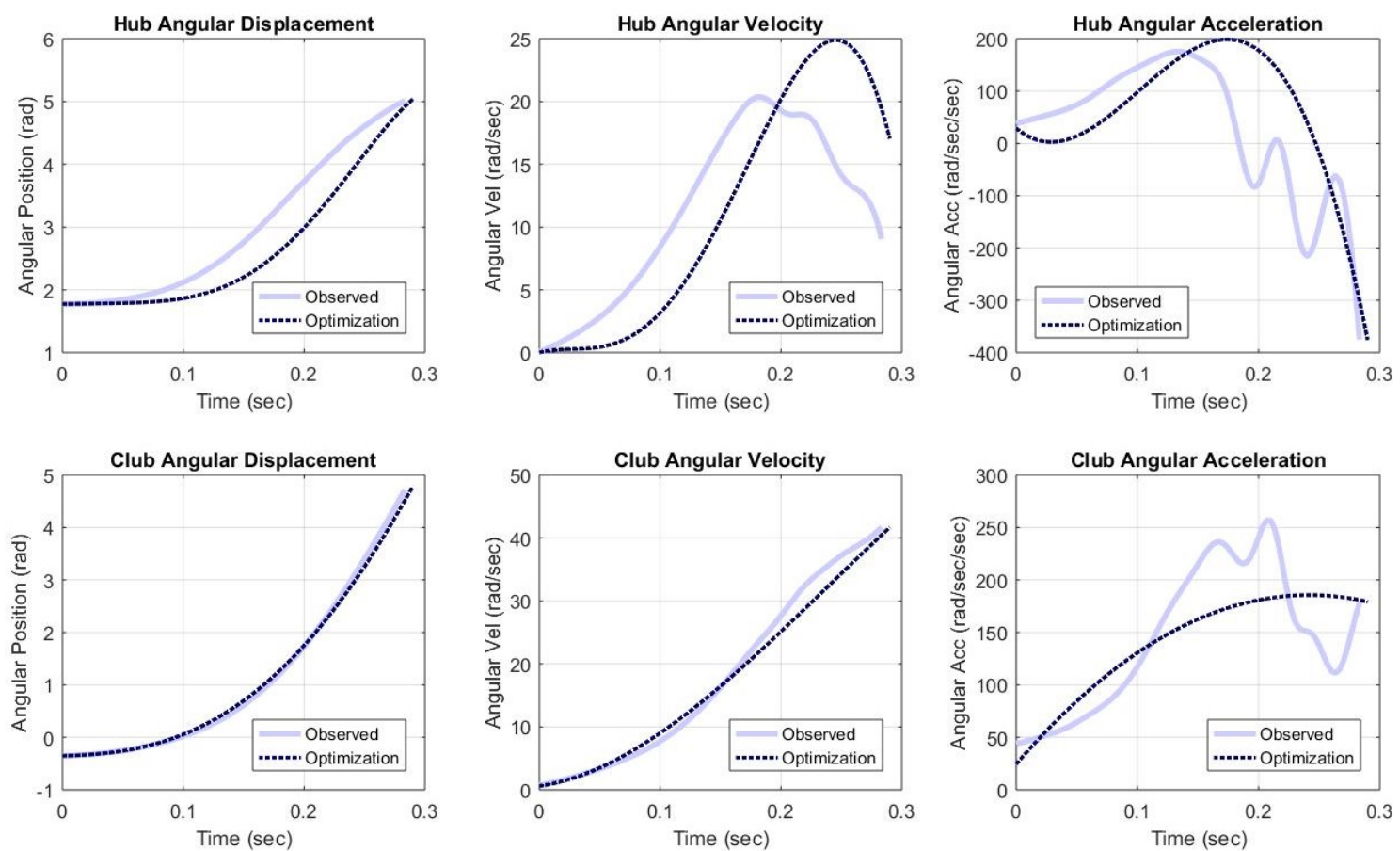

Figure 5. Participant 1 observed and optimised hub and club head trajectories.
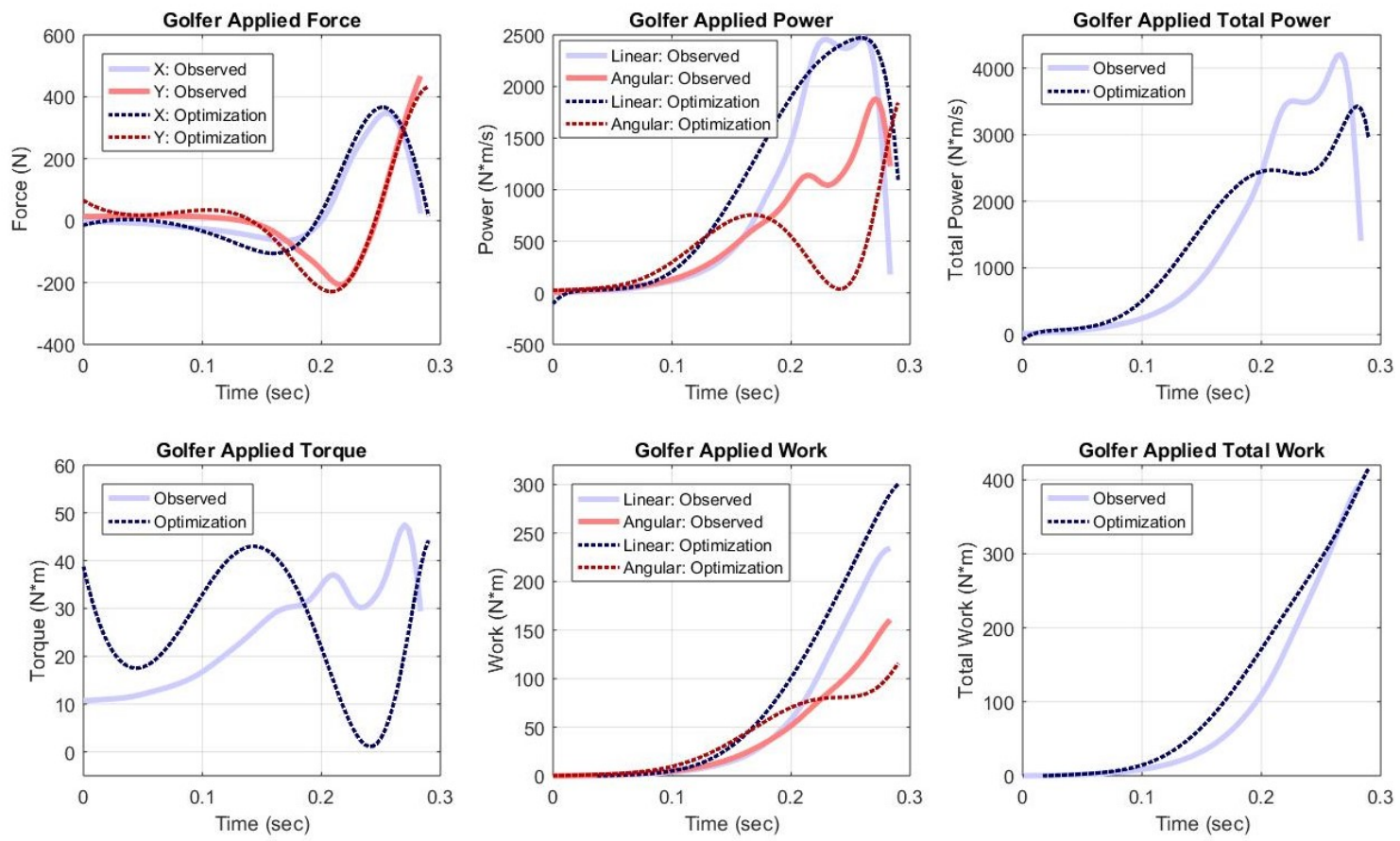

561 Figure 6. Participant 1 observed and optimised force, torque, power, and work responses. 

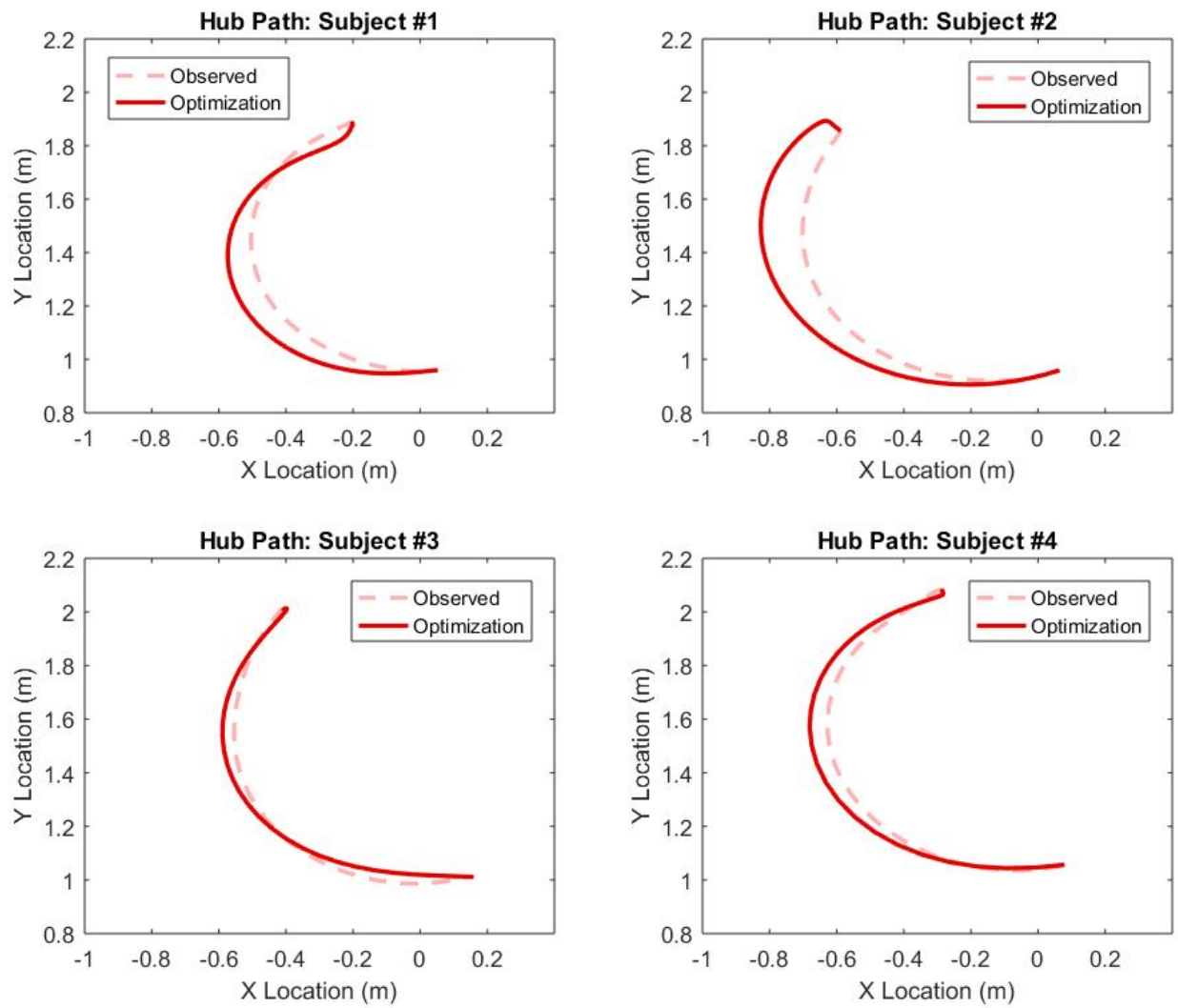

Figure 7. Participants 1-4 observed and optimised hub paths.

566

\section{SUPPLEMENTARY FIGURES}

Swing Motion - Original Qualisys Points

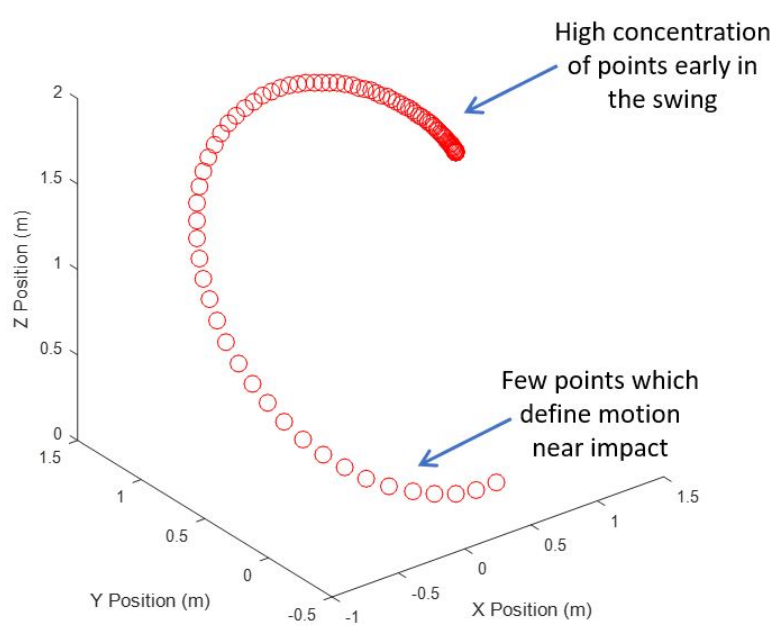

Swing Motion - Equidistant SplineFit Points

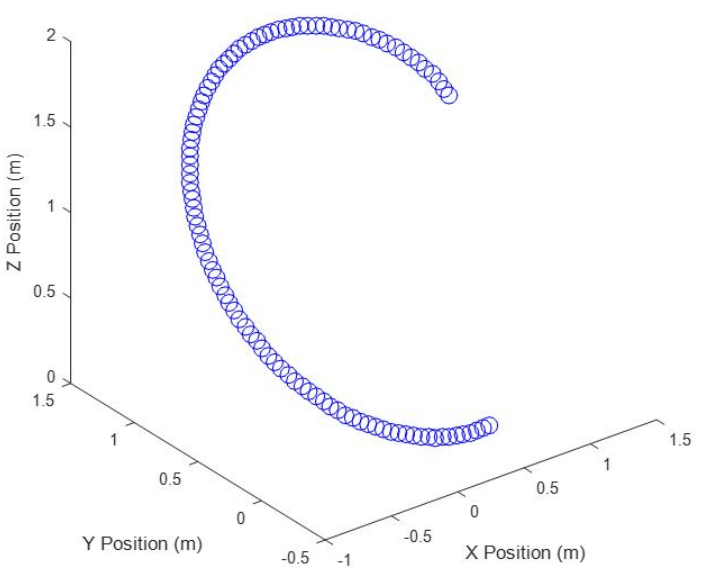

Supplementary Figure 1: Comparison between Participant 1 original club head path

570 (Left) and reconstruction with equidistance spline-fit points (Right). 

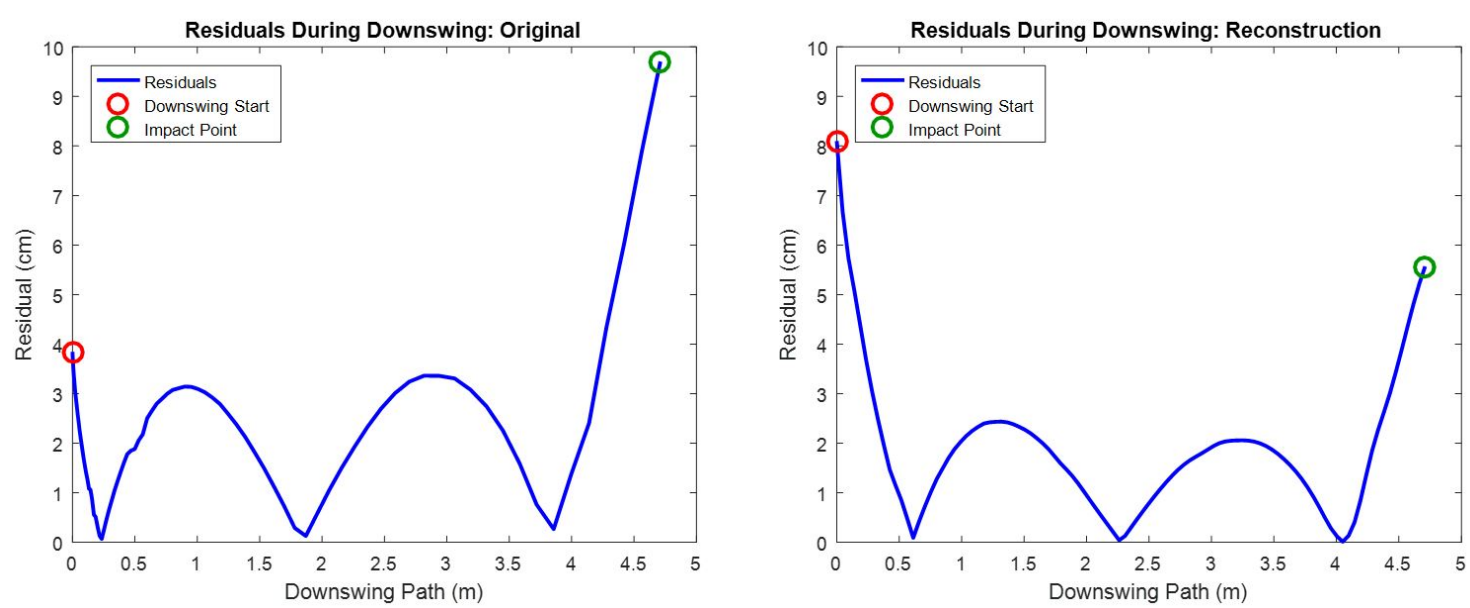

572

Supplementary Figure 2: RMSE comparison between Participant 1 original club head path and swing plane (Left) and reconstruction and swing plane (Right).

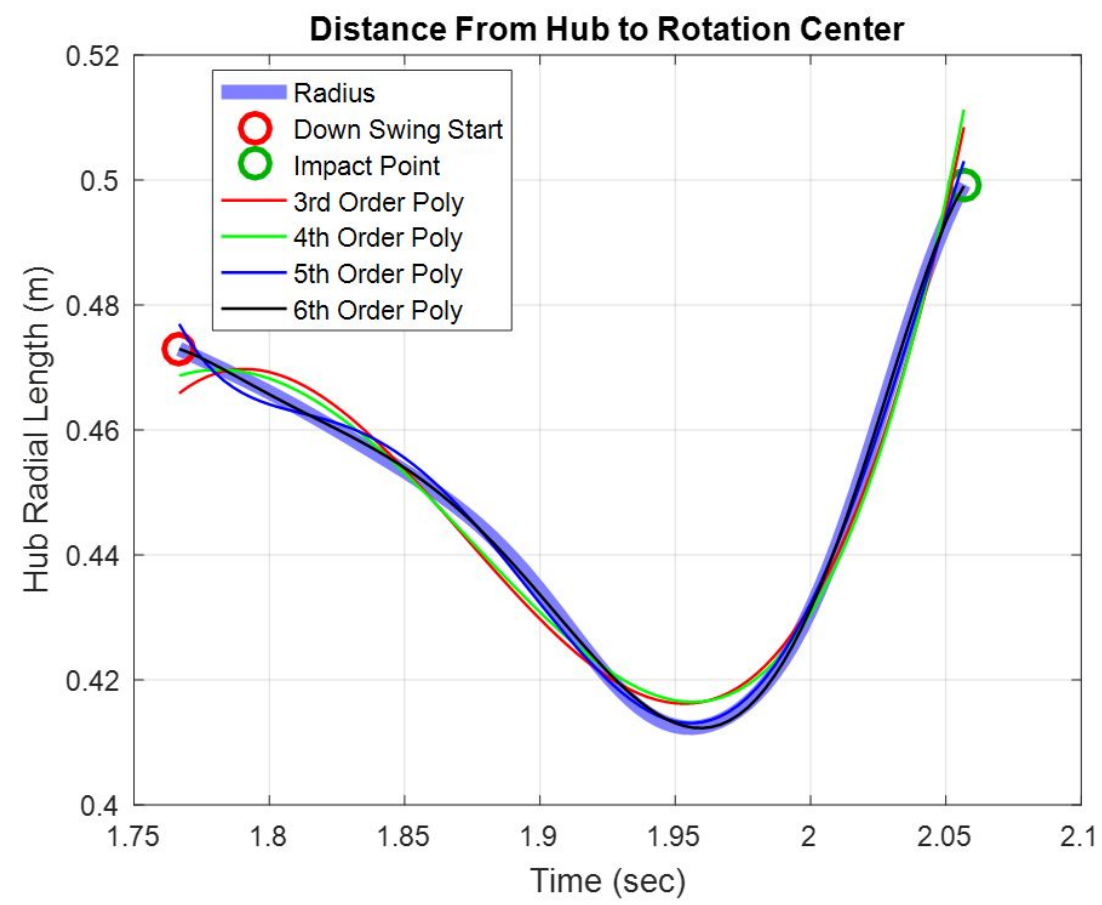

576
Supplementary Figure 3: Comparison between Participant 1 observed original hub radius length, expressed in time, and polynomial equations with different orders 


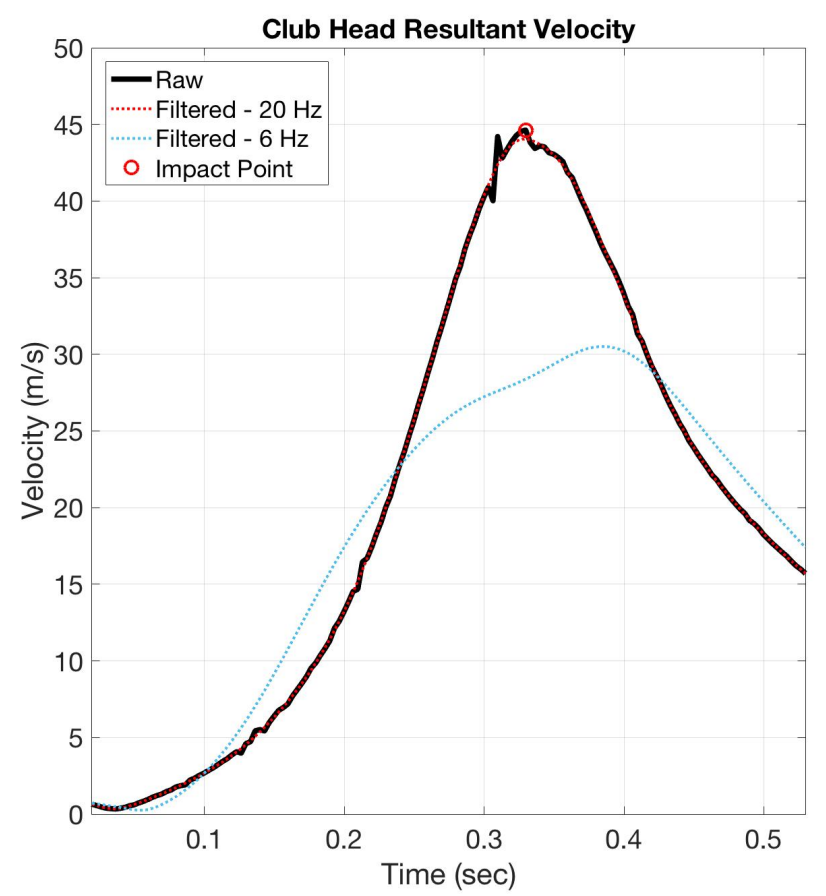

Supplementary Figure 4: Participant 1 club head path and the effects of filtering with 582 low frequencies

583 